\title{
Aplicação do modelo Return On Quality a clientes de uma empresa do setor metalúrgico
}

\section{(Applying Return on Quality Model: an analys on metallurgic company's clients)}

Sandra Maria da Silva sandra.m.amaral@hsbc.com.br Mestra pelo Programa de Pós-Graduação em Administração da Pontifícia Universidade Católica do Paraná - PPAD/PUC-PR Wesley Vieira da Silva wesley.vieira@pucpr.br Prof. Dr. do Programa de Pós-graduação em Administração da Pontifícia Universidade Católica do Paraná - PPAD/PUC-PR Paulo de Paula Baptista

p.baptista@pucpr.br

Prof. Dr. do Programa de Pós-graduação em Administração da Pontifícia Universidade Católica do Paraná - PPAD/PUC-PR

Editor Científico Responsável: Prof. Dr. Rubens da Costa Santos Professor no Mestrado em Hospitalidade na Universidade Anhembi Morumbi

Recebido em: 260/8/08

Aprovado em: 08/05/09

\begin{abstract}
Resumo
Neste estudo avalia-se a adequação do modelo Return on Quality (ROQ) para mensuração de retornos financeiros decorrentes de investimentos na melhoria da qualidade percebida. Os autores realizaram uma pesquisa de levantamento de caráter exploratório e descritivo com 120 clientes de uma empresa metalúrgica, selecionados por meio de amostragem aleatória simples. Os dados obtidos foram então submetidos às técnicas de Análise Fatorial, Análise de Cluster e Análise Discriminante. Os resultados obtidos mostraram que o Modelo ROQ pode ser uma ferramenta poderosa para os gestores de empresas no processo de tomada de decisões quanto à realização de investimentos. Os valores ROQ calculados apontaram para possibilidades de retornos bem acima do custo de capital da empresa, utilizado como parâmetro para cálculo do retorno financeiro.
\end{abstract}

Palavras-chave: Qualidade percebida. Retorno sobre a qualidade. Intenção de recompra.

\begin{abstract}
This study investigates the adequancy of Return on Quality (ROQ) model for measuring financial returns on perceived quality investments. The authors conducted an exploratory and descriptive survey with 120 clients of a metallurgic company. Those clients were selected by simple random sampling. Factorial Analyses, Cluster Analyses and Discriminate Analyses statistical techniques was used. The results showed that the ROQ Model can be a powerful tool for companies' managers make investments decisions. The ROQ calculated values pointed to higher payback standards than the capital cost of the company often used as a parameter for finance return calculation.
\end{abstract}

Key word: Perceived quality. Return over quality. Loyalty. 


\section{INTRODUÇÃO}

O avanço tecnológico e as mudanças sociais e políticas ocorridas nas últimas décadas trouxeram para as organizações o desafio de atuar em um mercado cada vez mais competitivo, com clientes cada vez mais exigentes. O desenvolvimento da internet e a queda dos regimentos socialistas são exemplos de como a tecnologia e as mudanças políticas contribuíram para formar o chamado "mercado globalizado".

$\mathrm{Na}$ época atual, marcada pelo mercado altamente competitivo e exigente, as técnicas de transações individuais e de comunicação em massa deram lugar à abordagem de relacionamento e à comunicação customizada. As ações do marketing passaram a ter como foco a aproximação entre o cliente e a empresa; índices como tempo de vida do cliente e satisfação passaram a fazer parte do processo de gestão, afinal "as organizações que conhecem melhor os seus clientes têm mais chance de mantê-los e, como consequência, de obter mais lucro por cliente em médio e longo prazo" (DANTAS, 2006, p. 11).

Apesar das inovações, a área de marketing ainda enfrenta um grande desafio, que é provar a eficiência de suas ações por meio da geração de retornos financeiros às empresas. Ao contrário de outras áreas tidas como funcionais, o trabalho realizado pela área de marketing nem sempre apresenta resultados diretos e que envolvam variáveis de fácil mensuração. Devido a isso, diversos estudos vêm sendo realizados com o objetivo de desenvolver técnicas para mensuração do retorno das ações de marketing. Entre esses estudos destacam-se os realizados por Anderson, Fornell e Lehmann (1994), Cover (1999), Ravald e Grönroos (1996), Mulhern (1999) e Morgan e Hunt (1994).

Dentre os métodos desenvolvidos para mensuração de desempenho, destaca-se o modelo Return on Quality (ROQ), desenvolvido pelos autores Rust, Zahorik e Keiningham (1994, 1995) e citado por Rust et al. (1999), além de Ribeiro et al. (2005).

O modelo ROQ parte do princípio de que os conceitos de satisfação e retenção ou intenção de recompra dos clientes, muito debatidos na área de marketing, apresentam como ponto de partida o desempenho da qualidade na prestação de serviços.

Ribeiro et al. (2005) demonstram a possibilidade de aplicação desse método de mensuração para avaliar o retorno financeiro dos investimentos em marketing, concluindo que esta constitui uma "ferramenta parcimoniosa e prática de avaliação do retorno dos investimentos associados à entrega de valor aos clientes, propiciando informações valiosas para os gerentes" (RIBEIRO et al., 2005, p. 14). 
Assim, este estudo tem por objetivo mensurar o retorno financeiro dos investimentos em qualidade percebida, sob a ótica do marketing de relacionamento, a partir da aplicação do modelo ROQ, utilizando como objeto de estudo uma instituição do setor da indústria metalúrgica que atua no segmento de reposição de peças para tratores utilizados na construção civil. Essa empresa está localizada na cidade de São José dos Pinhais - PR, pertencente à região metropolitana da capital do Estado, Curitiba.

Este artigo encontra-se estruturado em cinco seções que podem ser sumarizadas como se encontra a seguir: a primeira seção refere-se à parte introdutória da pesquisa; a segunda traz a fundamentação teórico-empírica, em que são apresentados conceitos concernentes à metodologia ROQ; a terceira enfoca a metodologia da pesquisa; a quarta traz a apresentação e a análise dos resultados; e a quinta faz as considerações finais e as recomendações para o desenvolvimento de futuras pesquisas.

\section{O MODELO DE PERFORMANCE EM MARKETING: RETURN ON QUALITY}

Partindo-se do objetivo de realizar uma aplicação do modelo ROQ em uma organização do setor da indústria metalúrgica, torna-se necessário realizar uma revisão de alguns conceitos que fazem parte dessa métrica. Assim, esta seção traz algumas considerações relacionadas à metodologia ROQ.

Mensurar o retorno das estratégias na área de marketing, tanto na abordagem de relacionamento quanto na abordagem transacional, é um desafio. Dentre os modelos existentes para mensuração de performance, Rust, Zahorik e Keiningham (1994, 1995) desenvolveram um modelo denominado Return On Quality, cujo principal objetivo é auxiliar a determinação de "em quais atributos do serviço investir para aumentar a percepção de valor do cliente, e proporcionar maior retorno financeiro" (RIBEIRO et al., 2005). 


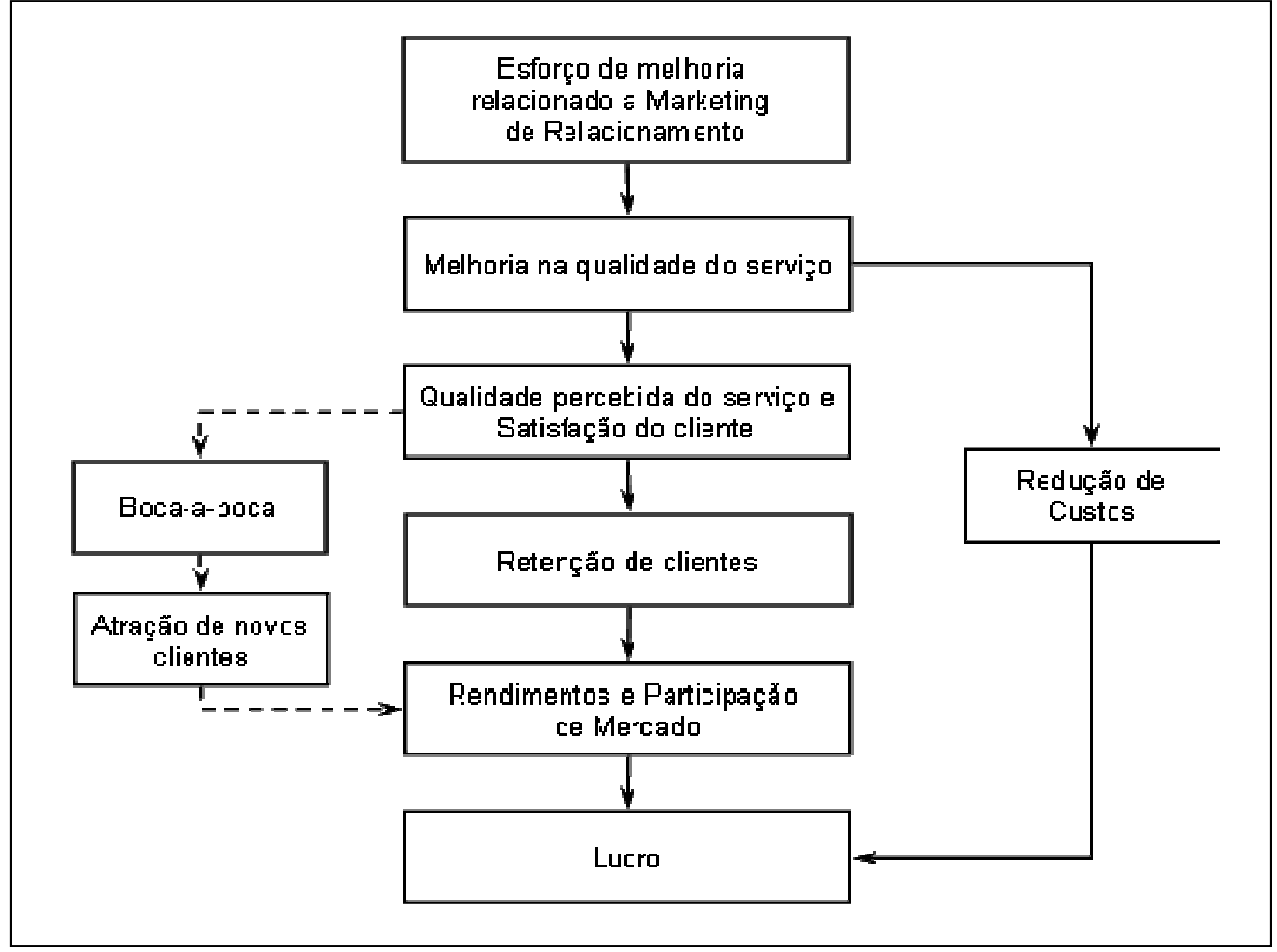

Figura 1: Modelo da melhoria na qualidade dos serviços e rentabilidade Fonte: RUST, ZAHORIK e KEININGHAM, 1995, p. 60.

O modelo ROQ, representado na Figura 1, prevê que o sucesso na realização de melhorias tem como consequência a melhoria na qualidade dos serviços prestados. A melhoria na qualidade dos serviços resulta em incremento dos níveis de qualidade percebida e de satisfação dos clientes, possibilitando ainda a redução de custos. $\mathrm{O}$ crescimento nos índices de satisfação de clientes aumenta os índices de retenção de clientes e possibilita maior divulgação por meio de propaganda boca-a-boca. Esta, por sua vez, aumenta a atração de novos clientes, que, aliada à maior taxa de retenção, possibilita maiores rendimentos e maior participação de mercado. Esse conjunto de resultados levaria ao incremento dos lucros obtidos pela organização.

Rust, Zahorik e Keiningham (1995) traduzem essa sequência de relações a partir de equações, expostas no Quadro 1. 


\begin{tabular}{|c|c|}
\hline Equação & Variáveis \\
\hline$A Q=f_{1}(X)+\varepsilon_{1}$ & $\begin{array}{l}\text { - } \quad \text { AQ é qualidade atual; } \\
\text { - } \quad X \text { é a variável que recebe os valores } 1 \text {, caso sejam } \\
\text { realizadas melhorias, e } 0 \text { se não forem realizadas } \\
\text { melhorias; } \\
\text { - } \varepsilon_{1} \text { é o erro aleatório do processo; }\end{array}$ \\
\hline$S=f_{2}(A Q, E)+\varepsilon_{2}$ & $\begin{array}{l}\text { - } S \text { é o vetor que mensura as atitudes, emoções, } \\
\text { percepções, qualidade percebida e satisfação do cliente; } \\
\text { - } E \text { é o vetor que contempla as expectativas do cliente e } \\
\text { outros fatores que influenciam } S ; \\
\text { - } \varepsilon_{2} \text { é o erro aleatório do processo; }\end{array}$ \\
\hline$C R=f_{3}(A Q)+\varepsilon_{3}$ & $\begin{array}{l}\text { - } \quad \text { CR é o vetor de redução de custos; } \\
-\quad \varepsilon_{3} \text { é o erro aleatório do processo; }\end{array}$ \\
\hline$R=f_{4}(S)+\varepsilon_{4}$ & $\begin{array}{l}-\quad R \text { representa a retenção de clientes; } \\
-\quad \varepsilon_{4} \text { é o erro aleatório do processo; }\end{array}$ \\
\hline$M S=f_{5}(R, M V)+\varepsilon_{5}$ & $\begin{array}{l}\text { - } \quad \text { MS é o vetor que reflete o desempenho do negócio; } \\
\text { - } \quad M V \text { é o vetor de todas as outras variáveis sistemáticas e } \\
\text { específicas de marketing que afetam a participação de } \\
\text { mercado; } \\
\text { - } \varepsilon_{5} \text { é o erro aleatório do processo; }\end{array}$ \\
\hline PROFIT $=f_{6}(M S, C R)+\varepsilon_{6}$ & $\begin{array}{l}\text { - } \quad \text { PROFIT é a medida que mensura o lucro; } \\
-\quad \varepsilon_{6} \text { é o erro aleatório do processo. }\end{array}$ \\
\hline
\end{tabular}

Quadro 1: Equações que traduzem o modelo de melhoria na qualidade dos serviços e rentabilidade Fonte: Adaptado de RUST, ZAHORIK e KEININGHAM, 1995, p. 60.

Para cálculo da participação de mercado de uma organização, Rust, Zahorik e Keiningham (1995) sugerem a utilização de diversos índices definidos a seguir e relacionados no Quadro 2:

- $N_{t}$ : tamanho do mercado no período t;

- $M_{t}$ : participação de mercado no período t;

- $C$ : cancelamentos, operacionalizado a partir do percentual de clientes que deixaram o mercado;

- $R$ : taxa de retenção de clientes da organização;

- $R^{\prime}$ : taxa de retenção de clientes da concorrência;

- $A$ : percentual de novos clientes que escolheram a organização. 


\begin{tabular}{|l|l|}
\hline \multicolumn{1}{|c|}{ Equação } & \multicolumn{1}{c|}{ Definição } \\
\hline (A) customers_retained $=R M_{t-1} N_{t-1}$ & $\begin{array}{l}\text { Clientes que permaneceram na } \\
\text { organização no tempo t-1 }\end{array}$ \\
\hline (B) customers_swicthing_to_us $=\left(1-R^{\prime}-C\right)\left(1-M_{t-1}\right) N_{t-1}$ & $\begin{array}{l}\text { Clientes que vieram de outras } \\
\text { organizações }\end{array}$ \\
\hline (C) new_customers $=A\left[C N_{t-1}+N_{t}-N t_{t-1}\right]$ & Novos clientes \\
\hline$\frac{(A)+(B)+(C)}{N_{t}}$ & $\begin{array}{l}\text { Participação de mercado da } \\
\text { organização }\end{array}$ \\
\hline
\end{tabular}

Quadro 2: Equações para cálculo da participação de mercado dentro do Modelo ROQ

Fonte: RUST, ZAHORIK e KEININGHAM, 1995, p. 61.

Por meio desses índices é possível observar que a função clientes retidos, definida como customer_retained, depende da taxa de retenção de clientes da empresa, da participação de mercado da empresa e do tamanho do mercado em questão.

A função cálculo dos clientes que vieram de outras organizações, ou seja, trocaram a concorrência pela empresa, definida por customer swicthing to us, depende da taxa de retenção da concorrência, do percentual de clientes que deixaram de operar no mercado, além dos índices de participação no mercado da empresa e do tamanho do mercado. Já a função para cálculo de novos clientes depende do percentual de clientes que deixaram de operar no mercado e do cálculo do tamanho do mercado. Finalizando essa sequência de índices, tem-se a participação de mercado da empresa, definida pela divisão da soma dos índices de retenção de clientes, do cálculo dos clientes que vieram de outras organizações e do cálculo de novos clientes pelo tamanho do mercado.

Da mesma forma, os autores apresentaram equações para cálculo do valor ROQ, a partir da determinação do Valor Presente Líquido, definido como NPV (Net Present Value). O NPV é o indicador utilizado para mensurar o retorno dos investimentos, dentro do Método ROQ. Nessas novas equações, torna-se necessária a definição de novos índices, estabelecidos a seguir:

- $G$ : taxa de crescimento de mercado constante;

- Y: preço médio e margem de contribuição constante;

- I: fator de desconto financeiro que reflete o custo de capital;

- X: estimativa da despesa realizada para obtenção da nova taxa de retenção;

- X: despesa total realizada;

- F: gasto de manutenção; 
- F0: despesa já realizada;

- F’: é um valor inicial de despesa que se assume para realizar as melhorias na qualidade de um processo específico do negócio, e que tenha sido identificado como importante.

A partir do Quadro 3 é possível identificar que o NPV é uma função que depende dos seguintes índices: o fator de desconto financeiro que reflete o custo de capital; o preço médio e a margem de contribuição constante; a participação de mercado; a taxa de crescimento de mercado constante; o tamanho de mercado; e a despesa realizada para obtenção da nova taxa de retenção.

\begin{tabular}{|l|l|}
\hline \multicolumn{1}{|c|}{ Equação } & \multicolumn{1}{c|}{ Definição } \\
\hline$N P V=\sum_{k=1}^{P}(1+I)^{-k}\left[Y M_{t+k}(1+G)^{k} N_{t}-X_{t+k}\right]$ & Valor Presente Líquido \\
\hline$N P V A S=F^{\prime}+\sum_{k=1}^{P}\left(F-F_{0}\right)(1+I)^{-k}$ & Valor Presente Líquido Adicional \\
\hline$R O Q=\frac{\left(N P V-N P V_{0}\right)}{N P V A S}$ & Valor ROQ \\
\hline
\end{tabular}

Quadro 3: Equações para cálculo do valor ROQ

Fonte: RUST, ZAHORIK e KEININGHAM, 1995, p. 61.

Percebe-se à luz do quadro supracitado que o índice NPVA depende do fator de desconto financeiro que reflete o custo de capital e do fator valor inicial de despesa necessário para realização dos esforços de qualidade. Dessa forma, tem-se o valor ROQ definido pela divisão da diferença entre os índices NPV e NPV inicial $\left(\mathrm{NPV}_{0}\right)$ pelo índice NPVAS.

Além das equações propostas para a mensuração da participação de mercado e para o cálculo dos valores NPV, NPAS e ROQ, os autores também sugerem fórmulas para cálculo dos índices de satisfação, qualidade e retenção de clientes.

Rust, Zahorik e Keiningham (1995) dividem o processo de aplicação do Modelo ROQ nas quatro etapas ilustradas na Figura 2. 


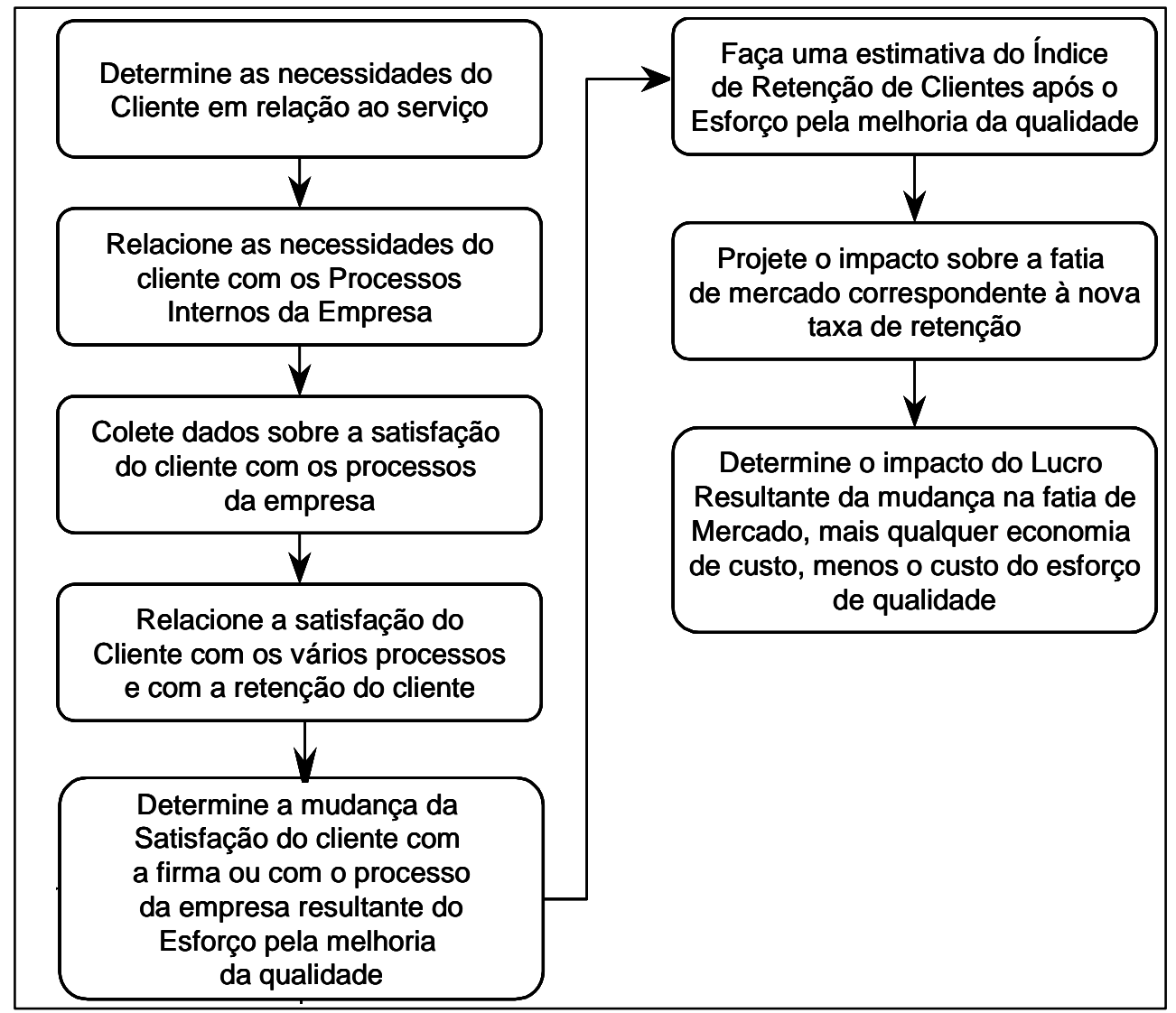

Figura 2: Etapas para determinação do retorno da qualidade (ROQ) Fonte: RUST, ZAHORIK e KEININGHAM, 1994, p. 9.

$\mathrm{Na}$ primeira etapa, uma pesquisa exploratória é utilizada para a determinação das necessidades dos clientes em relação aos produtos ou serviços prestados pela empresa. Posteriormente, essas necessidades são relacionadas com os processos internos da empresa que envolvem a fabricação do produto ou a prestação de serviços.

A etapa a seguir consiste na aplicação de uma pesquisa quantitativa que tenha como objetivo a determinação da satisfação dos clientes com os processos internos da empresa, sendo estes identificados na etapa anterior. Por meio da aplicação de técnicas estatísticas de análise, são identificados quais processos mais influenciam a satisfação e a retenção dos clientes da empresa.

A partir do estabelecimento de quais processos mais influenciam a satisfação e a retenção dos clientes, são realizadas mensurações para identificar a possível mudança da satisfação ou retenção dos clientes, mediante a realização de esforços que visem à melhoria dos processos. Com isso, é possível estabelecer o quanto as melhorias em qualidade podem impactar a satisfação ou retenção dos clientes.

Para finalizar, considerando-se uma situação extrema de retenção, pode-se estimar um 
novo percentual de participação do mercado. Ou seja, considerando-se que todos os esforços de melhorias sejam realizados com sucesso, a perda de clientes será menor, aumentando então a participação no mercado e a rentabilidade da empresa.

\section{METODOLOGIA}

Nesta pesquisa propõe-se a aplicar um modelo que tem como objetivo mensurar o retorno dos investimentos em qualidade percebida, sob a ótica do marketing de relacionamento, desenvolvido pelos autores Rust, Zahorik e Keiningham (1994, 1995), para uma aplicação real, no Brasil.

O delineamento de pesquisa adotado para este estudo foi um survey com perspectiva temporal transversal única, no qual a mensuração dos elementos ou variáveis ocorreu uma única vez no processo de investigação (HAIR JR. et al., 2005b).

Uma primeira etapa exploratória antecedeu a elaboração do questionário utilizado para realização da coleta de dados e aplicação do modelo ROQ. Essa etapa teve como objetivos entender e identificar as principais necessidades dos clientes em relação ao atendimento, aos produtos e aos serviços prestados pela Beta Metalúrgica Ltda. e, a partir das informações encontradas, elaborar o instrumento de coleta de dados a ser utilizado na etapa quantitativa da pesquisa.

Valendo-se da metodologia ROQ, a pesquisa quantitativa mensurou os índices de satisfação dos clientes com os serviços prestados e a intenção dos clientes em trocar ou deixar o fornecedor de serviços. Para mensuração da satisfação e da retenção dos clientes da Beta Metalúrgica Ltda., foram utilizadas escalas nominais e intervalares de mensuração.

\subsection{População e amostragem}

A população desta pesquisa corresponde ao conjunto de clientes da Beta Metalúrgica Ltda. que estavam cadastrados há mais de um ano e realizaram pelo menos duas compras de produtos da empresa até o mês de novembro de 2006, momento em que a amostra foi selecionada na base de cadastros da companhia. Ao final do processo de coleta de dados, 
validou-se uma amostra composta por 120 empresas clientes da Beta Metalúrgica Ltda., obtendo-se um erro amostral ou de estimação de aproximadamente $9 \%$ em nível de confiança de $95 \%$.

\subsection{Coleta de dados}

Para aplicação da pesquisa exploratória, realizaram-se 10 entrevistas em profundidade com clientes pertencentes à população-alvo, utilizando-se um roteiro com os principais tópicos a serem abordados no momento da entrevista. O roteiro seguiu a análise dos processos que envolvem os serviços e os produtos produzidos pela Beta Metalúrgica Ltda. As entrevistas realizadas na fase exploratória foram gravadas, para melhor acompanhamento dos resultados.

$\mathrm{Na}$ etapa quantitativa, um questionário elaborado a partir dos resultados encontrados na fase exploratória foi aplicado por meio de entrevistas telefônicas assistidas por computador (MALHOTRA, 2001).

Na pesquisa quantitativa são aplicadas, como técnicas estatísticas de análise de dados, a Análise Exploratória de Dados, Análise Cluster e Análise Discriminante (HAIR JR. et al., 2005b; MALHOTRA, 2001; CHARNET, 1999).

Conforme sugestão de Babbie (2001) para tratamento de dados de pesquisas do tipo levantamento ou survey design, são realizadas: análises univariadas, a partir da descrição do comportamento dos clientes em relação ao questionário aplicado e a partir de distribuições de frequências e estatísticas descritivas; análises bivariadas, que buscam verificar a existência de relações entre as variáveis analisadas, realizada a partir de coeficientes de correlação, distribuições comparativas de frequência e estatísticas descritivas comparativas; e análises multivariadas, que têm como objetivo determinar o impacto dos fatores que influenciam a satisfação e a intenção de recompra dos clientes.

Para cálculo do retorno financeiro dos investimentos em marketing de relacionamento, utilizou-se o indicador Valor Presente Líquido (VPL), conforme estipulado no Método ROQ. 


\section{APRESENTAÇÃO E ANÁLISE DOS RESULTADOS}

Nesta seção, a apresentação e a análise dos resultados da pesquisa são divididas em duas partes: a primeira é referente à análise dos dados da etapa qualitativa, enquanto a segunda refere-se à etapa quantitativa.

\subsection{Análise da etapa exploratória}

A partir da análise das respostas obtidas na fase qualitativa da pesquisa foram identificados dez diferentes atributos para a mensuração da qualidade dos serviços. Esses indicadores foram avaliados pelos pesquisadores e agrupados em quatro dimensões distintas, que são apresentadas no Quadro 4.

\begin{tabular}{|c|l|l|l|}
\hline $\begin{array}{c}\text { Variável } \\
\mathrm{X}_{1}\end{array}$ & \multicolumn{1}{|c|}{$\begin{array}{c}\text { Atributo } \\
\text { Agilidade no atendimento }\end{array}$} & $\begin{array}{l}\text { Descrição } \\
\text { Agilidade no processo de atendimento por parte da } \\
\text { equipe de vendas }\end{array}$ & $\begin{array}{c}\text { Dimensão } \\
\text { Atendimento }\end{array}$ \\
\hline $\mathrm{X}_{2}$ & $\begin{array}{l}\text { Autonomia dos } \\
\text { vendedores }\end{array}$ & $\begin{array}{l}\text { Autonomia dos vendedores no processo de } \\
\text { negociação }\end{array}$ & Atendimento \\
\hline $\mathrm{X}$ & Educação e cordialidade & Educação e cordialidade da equipe de vendas & Atendimento \\
\hline $\mathrm{X}_{3}$ & Forma de pagamento & Condições e formas disponíveis para pagamento & Cobrança \\
\hline $\mathrm{X}_{7}$ & Prazos de pagamento & Flexibilidade de formas de pagamento & Cobrança \\
\hline $\mathrm{X}_{4}$ & Disponibilidade de entrega & $\begin{array}{l}\text { Disponibilidade das linhas de produtos para pronta } \\
\text { entrega }\end{array}$ & Entrega \\
\hline $\mathrm{X}_{6}$ & Entrega dos produtos & O processo e a logística para entrega de produtos & Entrega \\
\hline $\mathrm{X}_{8}$ & Preço dos produtos & Preço dos produtos & Mix de produtos \\
\hline $\mathrm{X}_{9}$ & Qualidade dos produtos & $\begin{array}{l}\text { Qualidade dos produtos produzidos pela Beta } \\
\text { Metalúrgica Ltda. }\end{array}$ & Mix de produtos \\
\hline $\mathrm{X}_{10}$ & Variedade dos produtos & $\begin{array}{l}\text { Variedade da linha de produtos Beta Metalúrgica } \\
\text { Ltda. }\end{array}$ & Mix de produtos \\
\hline
\end{tabular}

Quadro 4: Atributos avaliados por meio da aplicação das escalas de importância e satisfação Fonte: Elaborado pelos autores do artigo.

Além dos atributos definidos a partir da pesquisa qualitativa, fez-se necessária a inclusão de indicadores para avaliação geral da empresa Beta Metalúrgica Ltda. Essa avaliação global foi realizada por meio de variáveis que buscavam identificar o atendimento 
das expectativas dos clientes e a satisfação geral destes com a empresa avaliada.

\subsection{Análise da etapa quantitativa}

No que tange ao uso da metodologia ROQ, a pesquisa quantitativa é realizada para se determinar a satisfação dos clientes e a probabilidade que estes apresentam de trocar de fornecedor, identificando possíveis melhorias na qualidade dos processos. Rust, Zahorik e Keiningham (1995) destacam que o principal ponto de interesse dos administradores é identificar em quais processos da empresa devem ser concentrados os esforços de melhorias, para que se obtenham, como resultados, uma maior satisfação e uma menor perda de clientes.

A primeira fase da análise quantitativa faz referência à Análise Descritiva e Exploratória de Dados, cujo objetivo é descrever os resultados univariados e relacionar a avaliação da satisfação versus a importância atribuída pelos clientes aos atributos avaliados. A segunda fase consiste na aplicação da Análise Fatorial nas variáveis que avaliam a satisfação dos clientes. O objetivo da aplicação dessa técnica é testar a hipótese de que a satisfação é formada por um construto unidimensional.

A terceira fase da análise da etapa quantitativa é especificamente voltada para aplicação da metodologia ROQ. Nessa fase, são determinados os pesos das variáveis que mais contribuem com a satisfação dos clientes e daquelas que mais contribuem com a intenção de recompra dos clientes.

\subsection{Análise descritiva e exploratória de dados}

A Análise Descritiva e Exploratória dos Dados é realizada por meio da distribuição de frequências de cada uma das variáveis presentes no questionário e do cruzamento, a partir da construção de uma matriz, das avaliações dos graus de satisfação e de importância mediante a avaliação dos atributos (variáveis) definidos na etapa qualitativa. Rust, Zahorik e Keiningham (1994, 1995) definem a matriz de cruzamento dessas variáveis como mapeamento da importância-desempenho.

Os graus de satisfação e de importância foram avaliados por meio da aplicação de duas 
PUC Minas

E\&G - REVISTA ECONOMIA E GESTÃO

ISSN 1984-6606

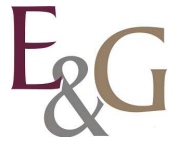

escalas intervalares de cinco pontos. Os atributos (variáveis) avaliados por meio da aplicação dessas escalas, definidos na etapa qualitativa, estão descritos no Quadro 4, já apresentado.

Os resultados do mapeamento importância-desempenho podem ser visualizados na Figura 3, na qual se observa que o atributo qualidade dos produtos é o ponto forte da Beta Metalúrgica Ltda. Esse atributo, além de ser considerado como o mais importante por parte dos clientes, é também o de melhor avaliação ao considerar-se o Índice de Encantamento do Cliente.

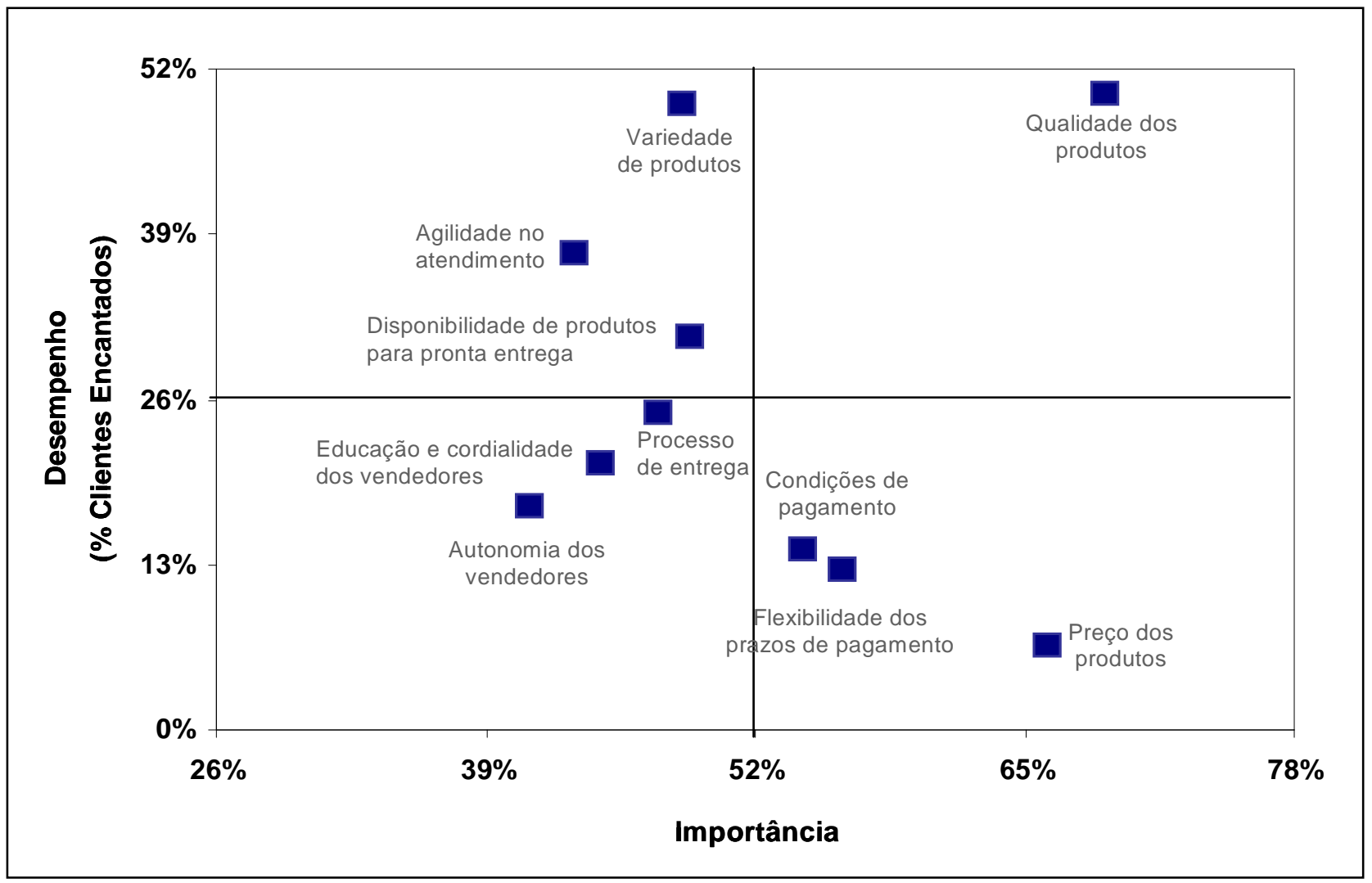

Figura 3: Mapeamento importância-desempenho baseada no questionamento direto dos clientes Fonte: Elaborado pelos autores do artigo.

Como pontos fracos, destacam-se os atributos "preços dos produtos", "flexibilidade nos prazos de pagamento" e "condições ou formas disponíveis para pagamento dos produtos". Esses atributos, embora apresentem um alto Índice de Excelência em Importância, apresentam um baixo percentual de clientes muito satisfeitos.

Os atributos, "variedade da linha de produto", "agilidade no processo de atendimento por parte da equipe de vendas" e "disponibilidade da linha de produtos para pronta entrega", embora apresentem um alto Índice de Encantamento do Cliente, não estão entre os atributos considerados como mais importantes. Já os atributos "educação e cordialidade da equipe de vendas", "autonomia dos vendedores no processo de negociação" e "processo e logística de 
entrega dos produtos" apresentam os menores valores tanto para os índices de satisfação quanto para os de importância.

\subsection{Aplicação da técnica de análise fatorial}

Após a realização da análise descritiva e exploratória dos dados, procedeu-se a uma análise fatorial com o objetivo de identificar se a satisfação dos clientes da empresa Beta Metalúrgica Ltda. pode ou não ser descrita a partir de um construto unidimensional.

A análise fatorial é aplicada nos resultados das avaliações, por parte dos clientes, das variáveis $X_{1}$ até $X_{10}$. Essas variáveis procuraram mensurar a satisfação dos clientes da Beta Metalúrgica Ltda. nos diversos processos de atendimento, produtos e serviços. As avaliações são realizadas por meio da aplicação de uma escala intervalar de cinco pontos.

A partir dos resultados auferidos da Análise Fatorial observou-se que a satisfação não pode ser representada por meio de um construto unidimensional. Entre os 10 atributos avaliados, nove foram considerados significativos e formaram três dimensões de avaliações apresentadas na Tabela 1. Apesar disso, para a próxima etapa são considerados os desempenhos individuais das variáveis, e não os escores fatoriais de cada dimensão.

A consistência interna dos resultados encontrados com a aplicação dessa escala é analisada por meio da aplicação do coeficiente Alfa de Cronbach, como evidencia a Tabela 1. Hair Jr. et al. (2005a, p. 90) definem o coeficiente Alfa de Cronbach como uma "medida de confiabilidade que varia de 0 até 1 , sendo os valores de 0,60 até 0,70 considerados o limite inferior de aceitabilidade". Esse coeficiente é utilizado para avaliar o grau de consistência entre as variáveis de uma escala múltipla. 
Tabela 1

Análise fatorial e Coeficiente Alfa de Cronbach

\begin{tabular}{|c|c|c|c|c|}
\hline Variável & Variável & $\begin{array}{c}\text { Produtos e } \\
\text { Entrega }\end{array}$ & $\begin{array}{c}\text { Negociação } \\
\text { preço }\end{array}$ & $\begin{array}{l}\text { Agilidade } \\
\text { Atendimento }\end{array}$ \\
\hline $\mathrm{X}_{4}$ & Disponibilidade das linhas de produtos para pronta entrega & 0,715 & & \\
\hline $\mathrm{X}_{6}$ & O processo e a logística de entrega dos produtos & 0,580 & & \\
\hline $\mathrm{X}_{9}$ & Qualidade dos produtos produzidos pela Beta Metalúrgica Ltda. & 0,880 & & \\
\hline $\mathrm{X}_{10}$ & Variedade da linha de produtos da Beta Metalúrgica Ltda. & 0,918 & & \\
\hline $\mathrm{X}_{2}$ & Autonomia dos vendedores no processo de negociação & & 0,714 & \\
\hline $\mathrm{X}_{3}$ & Condições ou forma disponíveis para pagamento dos produtos & & 0,741 & \\
\hline $\mathrm{X}_{7}$ & Flexibilidade dos prazos de pagamento & & 0,755 & \\
\hline $\mathrm{X}_{8}$ & Preços dos produtos & & 0,636 & \\
\hline $\mathrm{X}_{1}$ & Agilidade no processo de atendimento por parte da equipe de vendas & & & 0,636 \\
\hline \multicolumn{2}{|r|}{ Variância explicada \% } & 28,47 & 21,68 & 12,95 \\
\hline \multicolumn{2}{|c|}{ Alfa de Cronbach } & 0,821 & 0,697 & - \\
\hline
\end{tabular}

Fonte: Elaborado pelos autores do artigo.

Foram aplicados, ainda, os testes de Esfericidade de Bartlett e o teste de adequação da amostra reportado como KMO, cujos resultados validaram a aplicação da análise fatorial. Os resultados dos testes aplicados encontram-se na Tabela 2.

Tabela 2

Teste de Bartlett e KMO

\begin{tabular}{l|l|c}
\hline \multicolumn{2}{l|}{ Medida de adequação da amostra Kaiser-Meyer-Olkin (KMO) } & 0,738 \\
\hline \multirow{2}{*}{ Teste de Esfericidade de Bartlett } & Qui-Quadrado aproximado & 476,177 \\
& Gl & 45 \\
& Significância & 0,000 \\
\hline
\end{tabular}

Fonte: Elaborado pelos autores do artigo.

\subsection{Mensuração da Importância Relativa por meio da Análise de Cluster e da Análise Discriminante}

A próxima fase da análise quantitativa faz referência à estimação da importância das variáveis na qual a empresa Beta Metalúrgica Ltda é avaliada, estimando suas contribuições para a satisfação geral dos clientes e para a intenção de recompra destes. Para determinar a importância dos processos avaliados, a técnica utilizada é a Análise Discriminante, aplicada para duas variáveis respostas, uma referente à satisfação dos clientes e outra referente à intenção de recompra destes. 
- Importância Relativa das variáveis para satisfação geral

Os resultados da Análise de Cluster identificaram a existência de dois grupos, denominados os clientes menos satisfeitos e os clientes mais satisfeitos. O processo de escolha de dois clusters deu-se mediante a análise de ferramentas como o gráfico dendrograma e a análise das diferenças entre as médias e proporções para cada uma das variáveis, considerando-se a formação de cinco até dois clusters.

Para esta pesquisa, utiliza-se o Índice de Potência da Análise Discriminante para determinação da importância de cada processo. Hair Jr. et al. (2005b) apontam que o Índice de Potência é utilizado para comparar a discriminação geral de cada variável independente para todas as Funções Discriminantes Formadas e ele representa o poder discriminatório de cada variável.

Assim, apesar de nesta análise ser verificada a existência de apenas uma Função Discriminante, o Índice de Potência é estimado apenas para as variáveis significativas nessa função, como forma de se encontrar o percentual ponderado que representa a importância relativa de cada variável do modelo discriminante. As estimativas do Índice de Potência e da Importância Relativa encontram-se evidenciadas na Tabela 3. 
Tabela 3

Estimação da importância relativa das variáveis para a satisfação dos clientes

\begin{tabular}{c|c|c|c|c|c}
\hline Variáveis & Carga da & Carga ao & Autovalor da & Índice de & Importância \\
\hline Independentes & função & Quadrado & Função & $\begin{array}{c}\text { Potência } \\
\mathbf{( 1 )}\end{array}$ & Relativa (2) \\
\hline$X_{2}$ & 0,596 & 0,355 & 1,673 & 0,594 & $33 \%$ \\
$X_{3}$ & 0,487 & 0,237 & 1,673 & 0,397 & $22 \%$ \\
$X_{4}$ & 0,187 & 0,035 & 1,673 & 0,059 & $3 \%$ \\
$X_{6}$ & 0,25 & 0,063 & 1,673 & 0,105 & $6 \%$ \\
$X_{8}$ & 0,479 & 0,229 & 1,673 & 0,384 & $21 \%$ \\
$X_{9}$ & 0,396 & 0,157 & 1,673 & 0,262 & $15 \%$ \\
\hline
\end{tabular}

- Índice de Potência Estimado = carga da função ao quadrado $x$ autovalor da função

- Importância Relativa Estimada = ponderação entre o Índice de Potência Estimado para cada variável e a soma total dos Índices de Potência Estimados para todas as variáveis significativas para o modelo.

Fonte: Elaborado pelos autores do artigo.

Logo, considerando-se a satisfação dos clientes da Beta Metalúrgica Ltda., a variável de maior importância relativa é a autonomia dos vendedores no processo de negociação, seguida pelas variáveis condições ou formas disponíveis para pagamento dos produtos, preços dos produtos e qualidade dos produtos. As variáveis referentes à entrega dos produtos $\left(\mathrm{X}_{4} \mathrm{e}\right.$ $\mathrm{X}_{6}$ ) apresentaram a menor importância relativa quando considerada a satisfação dos clientes.

- Importância Relativa das Variáveis para Intenção de Recompra dos Clientes

Após a estimação da importância relativa das variáveis que mais contribuem para discriminar o comportamento dos clientes quanto à satisfação com os processos de atendimento, produtos e serviços prestados pela empresa Beta Metalúrgica Ltda., o próximo passo da análise quantitativa consiste em determinar a importância relativa das variáveis que mais impactam a intenção de recompra desses clientes.

Conhecendo-se a importância relativa e o percentual de clientes encantados de cada variável, é possível construir o mapeamento importância-desempenho sugerido por Rust, Zahorik e Keiningham (1994, 1995). Por meio do mapeamento, presente na Figura 4, é possível observar que as prioridades da empresa Beta Metalúrgica Ltda. são os investimentos que possibilitem a redução dos preços de seus produtos e mudanças estratégicas que forneçam maior autonomia para sua equipe de vendas, no processo de negociação. 


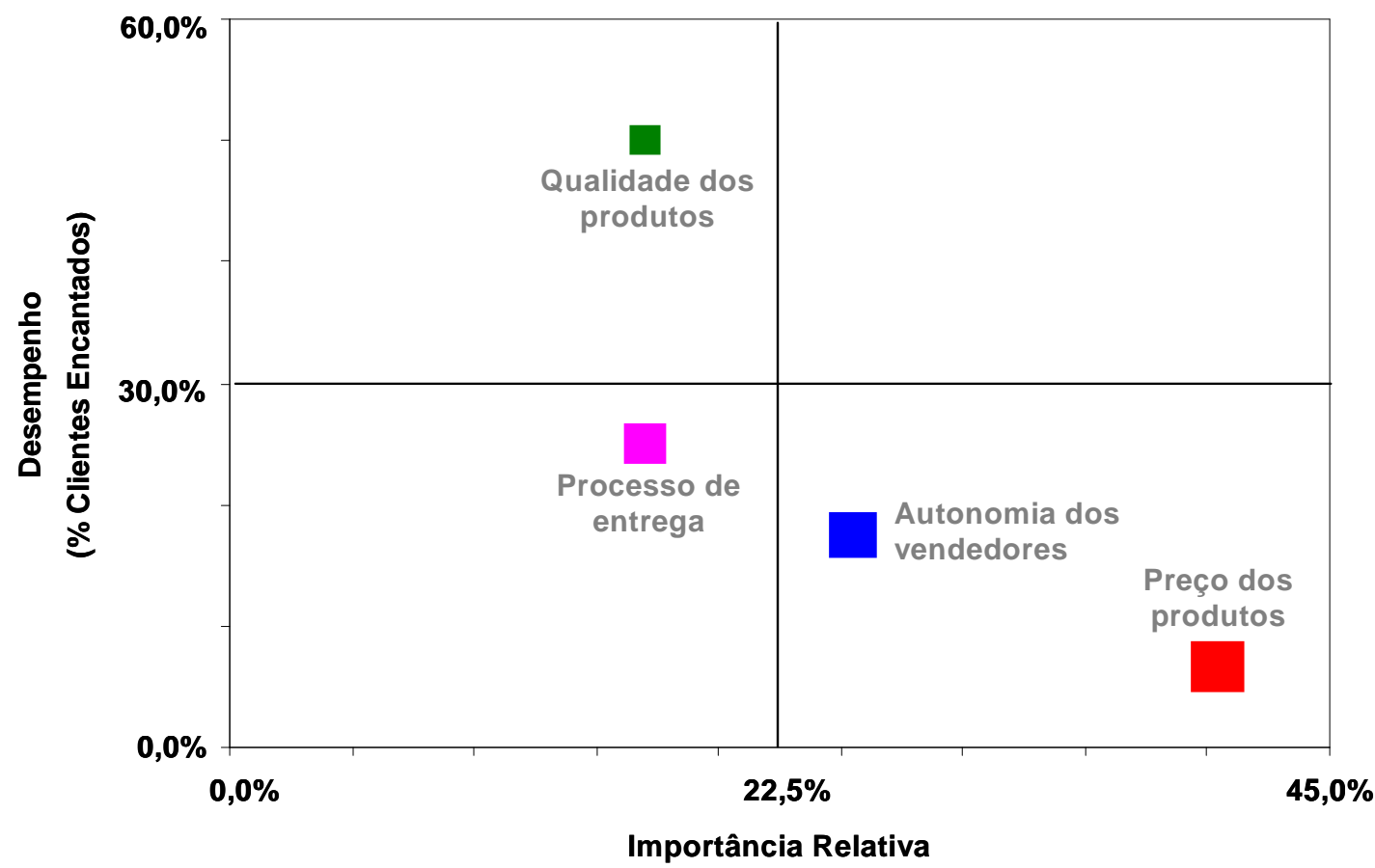

Figura 4: Mapeamento importância-desempenho - classificação dos clientes quanto a intenção de recompra Fonte: Elaborado pelos autores do artigo.

Quanto à qualidade de seus produtos, os resultados mostram que este é um ponto forte da empresa e que deve procurar ser mantido, ou seja, as possíveis mudanças referentes ao preço não podem prejudicar a qualidade dos produtos. Já o processo de entrega pode ser colocado, nesse momento, como última prioridade, entre as quatro variáveis selecionadas pelo modelo, para realização de melhorias.

Dentro desse processo, o efeito do encantamento do cliente é calculado para o grupo de clientes classificados com a alta intenção de recompra. O cruzamento entre as variáveis satisfação geral e os clusters formados se mostra significante estatisticamente, conforme aplicação dos testes de Kruskal-Wallis e Qui-quadrado.

Tabela 4

Efeito do encantamento

\begin{tabular}{l|c}
\hline Satisfação Geral & $\begin{array}{c}\text { Alta intenção de } \\
\text { Recompra }\end{array}$ \\
\hline Insatisfeitos & $0 \%$ \\
Meramente Satisfeitos & $47 \%$ \\
Encantados & $75 \%$ \\
Efeito Encantamento & $\mathbf{2 8 \%}$ \\
\hline
\end{tabular}

Fonte: Elaborado pelos autores do artigo. 
Os resultados da Tabela 2 mostraram que o impacto da classificação de clientes com a alta intenção de recompra, quando um cliente passa de meramente satisfeito para encantado, é igual a $28 \%$.

\subsection{Definição estratégica e o valor ROQ}

A partir dos resultados apresentados, o próximo passo da aplicação do modelo ROQ consiste em desenvolver estratégias que viabilizem a realização dos esforços em qualidade nas variáveis que impactam diretamente a satisfação e a intenção de recompra dos clientes da empresa Beta Metalúrgica Ltda. Além dessa definição, é necessário ainda estimar os custos envolvidos na aplicação das estratégias desenvolvidas, para cálculo do valor do ROQ.

Assim, esta seção está divida em duas subseções, sendo a primeira referente às estratégias a serem desenvolvidas pela organização e a segunda referente ao valor do ROQ.

\subsubsection{Definição de estratégias de investimentos em melhorias do processo}

Os resultados encontrados na pesquisa quantitativa mostram que os pontos que mais impactam a intenção de recompra dos clientes da Beta Metalúrgica Ltda. fazem referência ao preço de seus produtos e à autonomia de seus vendedores no processo de negociação.

Embora os resultados encontrados apontem para o desenvolvimento de estratégias que melhorem a satisfação dos clientes quanto ao preço, à entrega e à negociação, não são trabalhadas, nesta pesquisa, estratégias voltadas ao processo de entrega dos produtos. Essa situação se dá devido ao posicionamento atual da empresa, que optou por não realizar alterações no processo de entrega de seus produtos. Dessa forma, as principais estratégias elaboradas para realização de melhorias na empresa visam atingir a redução viável dos preços dos produtos e uma maior percepção de flexibilidade na negociação e autonomia dos vendedores.

Esses dois pontos fazem emergir as sugestões de cinco estratégias de investimentos para a Beta Metalúrgica Ltda. A Tabela 3 contém um resumo das estratégias propostas e os valores de investimentos envolvidos nesse processo. 
Tabela 5

Estratégias desenvolvidas para aplicação do Modelo ROQ

\begin{tabular}{l|l|c|c}
\hline \multicolumn{1}{c|}{ PONTO } & \multicolumn{1}{|c|}{ ESTRATÉGIA } & DURAÇÃO & INVESTIMENTO \\
\hline $\begin{array}{l}\text { Redução viável dos } \\
\text { preços dos produtos }\end{array}$ & $\begin{array}{l}\text { (1) Comitê para avaliação dos } \\
\text { processos de produção } \\
\text { (2) Programa melhores ideias } \\
\text { (3) Melhores preços para matéria- } \\
\text { prima } \\
\text { (4) Melhor qualificação da equipe de } \\
\text { vendas }\end{array}$ & $\begin{array}{l}\text { 6 meses } \\
6 \text { meses }\end{array}$ & $\mathrm{R} \$ 15.800,00$ \\
$\begin{array}{l}\text { Maior percepção de } \\
\text { flexibilidade na } \\
\text { negociação e } \\
\text { autonomia dos } \\
\text { vendedores }\end{array}$ & $\begin{array}{l}\text { (5) Segmentar a carteira de clientes por } \\
\text { meio da adoção do modelo RFV }\end{array}$ & 1 ano & $\mathrm{R} \$ 2.00,00$ \\
\hline INVESTIMENTO TOTAL & $\mathrm{R} \$ 6.000,00$ \\
\hline
\end{tabular}

Fonte: Elaborado pelos autores do artigo.

\subsubsection{Mensuração do Valor Return on Quality}

A partir da definição das estratégias e do investimento necessário para aplicação destas, o passo final da aplicação do modelo ROQ consiste em calcular o valor ROQ. No exemplo de aplicação do modelo ROQ, o retorno financeiro encontrado para a rede de hotéis Nacional foi de $44,6 \%$, sendo esse percentual de retorno avaliado como um retorno muito saudável (RUST; ZAHORIK; KEININGHAM, 1995).

O cálculo do valor ROQ é feito mediante a aplicação das equações, presentes no Quadro 5, para cálculo dos valores NPV, $\mathrm{NPV}_{0}$ e NPVAS. Para isso, é necessário definir as seguintes variáveis que compõem essas equações: taxa de crescimento de mercado, margem média de contribuição por cliente, custo de capital, participação de mercado e valor total da despesa para realização dos investimentos.

Como taxa de crescimento de mercado, adotou-se o valor estipulado pelo IBGE para crescimento da indústria de bens de capital em 2005, com um valor estimado em torno de 5,7\%. A margem de contribuição por cliente e o custo de capital foram obtidos na Beta Metalúrgica Ltda., sendo estes, respectivamente, de $\mathrm{R} \$ 148,00$ e uma taxa estimada de 3\%. O valor das despesas para investimentos, conforme a Tabela 5, é igual a $\mathrm{R} \$ 33.976,00$. As despesas foram dividas em $20 \%$ para despesa inicial e $80 \%$ para distribuição como despesas mensais.

Como participação de mercado (market share), é utilizado o valor de 0,50\%, obtido, 
de forma empírica, dos sócios proprietários da empresa. O tamanho do mercado é estimado por meio da aplicação direta do percentual de market share em relação ao número de clientes da Beta Metalúrgica Ltda. O período de análise corresponde ao tempo para realização das estratégias, que é de doze meses.

Vale ressaltar que, na ausência de valores oficiais, os valores expressos anteriormente são estimativas que visam à aplicabilidade do modelo ROQ. Assim, dado o comportamento dos clientes da Beta Metalúrgica Ltda. quanto à intenção de recompra, o modelo ROQ é aplicado levando-se em consideração dois cenários. Esses cenários são formados a partir da expectativa de aumento da base de clientes da Beta Metalúrgica Ltda. e da expectativa de aumento da margem de contribuição por cliente.

No cenário 1 são consideradas as expectativas de aumento de $5 \%$ da base de clientes e de 5\% na margem média de contribuição por cliente. Partindo desses valores, o valor ROQ é igual a $68 \%$. Para o cenário 2 é considerada apenas a expectativa de aumento da base de clientes, sendo o valor ROQ igual a 33\%. Da mesma forma, considerando-se a hipótese de perda de clientes, ou seja, diminuição do market share, o valor ROQ simulado passa a ser negativo. A comparação entre os resultados positivos encontra-se na Figura 5.

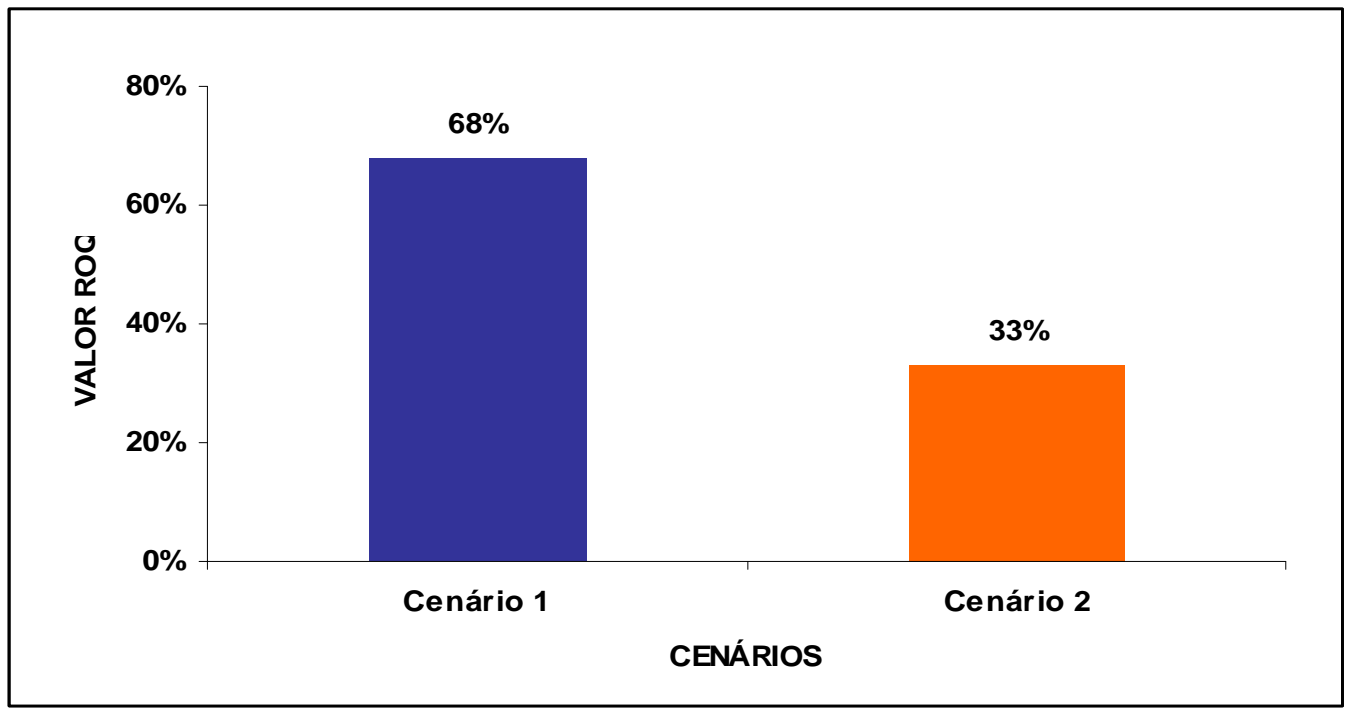

Figura 5: Valor ROQ calculado mediante a proposição de dois cenários Fonte: Elaborado pelos autores do artigo

Assim, partindo-se da aplicação do modelo ROQ, tem-se que os esforços de melhorias, estabelecidos por meio da análise do impacto da avaliação da satisfação sobre a intenção de recompra dos clientes, podem trazer um retorno significativo para a Beta Metalúrgica Ltda. 
Dentre os resultados encontrados, calculados a partir da elaboração de dois cenários, percebe-se que o valor ROQ aponta para um retorno positivo, quando considerada a expectativa de aumento da base de clientes, e para um retorno negativo, quando considerada a hipótese de perda de clientes. As variações entre os resultados são esperadas à medida que o método tem como base o aumento do market share e, consequentemente, o aumento do lucro da organização, mediante a realização de esforços de melhorias na qualidade dos processos e serviços.

Os resultados positivos encontrados nos dois cenários, $68 \%$ e $33 \%$ respectivamente, são expressivos quando comparados ao valor ROQ, igual a 44,6\%, calculado na avaliação da rede de hotéis Nacional, no exemplo utilizado pelos autores Rust, Zahorik e Keiningham (1995). Comparando-se os resultados, pode-se fazer uma analogia ao exemplo dos autores, que avaliam como muito saudável o valor encontrado para a rede de hotéis Nacional. Dessa forma, tanto para o cenário 1 quanto para o cenário 2, pode-se dizer que as ações de melhorias propostas nesta pesquisa trarão um retorno muito saudável para a empresa Beta Metalúrgica Ltda.

Da mesma forma, os resultados encontrados são comparados ao custo de capital da empresa, que, no modelo ROQ, substitui a TMA - Taxa Mínima de Atratividade, ou seja, o valor mínimo que se deseja ganhar na realização de um investimento. Quando comparado ao custo de capital informado pela empresa (3\%), os retornos obtidos pelo valor ROQ, aplicado aos dois cenários, são expressivamente melhores.

A comparação entre os valores calculados nesta pesquisa e obtidos pelos autores Rust, Zahorik e Keiningham (1995) encontra-se na Figura 6.

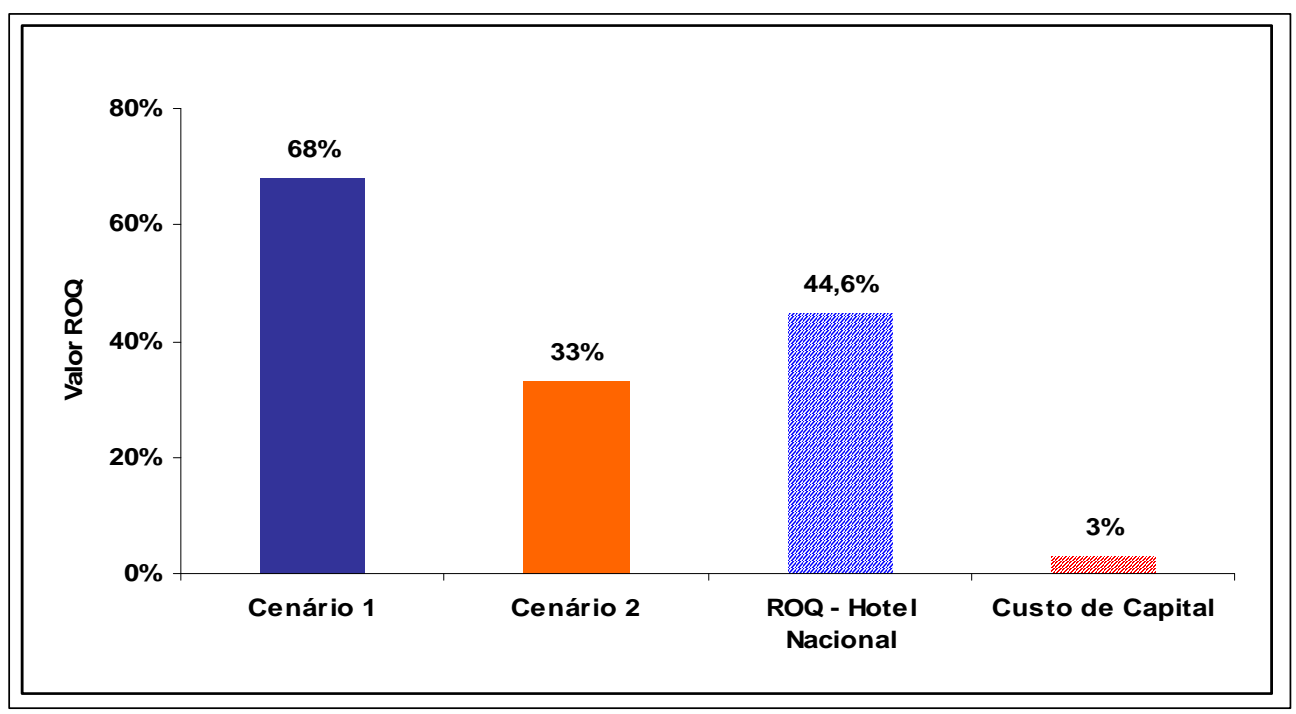

Figura 6: Comparação entre o Valor ROQ calculado mediante a proposição de dois cenários e o Valor 
Fonte: Elaborado pelos autores do artigo.

Assim, partindo-se da aplicação de um modelo teórico, os resultados encontrados vão ao encontro das necessidades práticas das empresas atuais, que são a busca por maior eficiência e maior aproximação aos clientes e, consequentemente, maior lucro, devido às mudanças ocorridas no mercado nos últimos anos.

\section{CONSIDERAÇÕES FINAIS, LIMITAÇÕES E RECOMENDAÇÕES}

Esta pesquisa teve como objetivo geral mensurar o retorno financeiro dos investimentos em qualidade percebida, sob a ótica do marketing de relacionamento, em uma instituição do setor da indústria metalúrgica, pautando-se no uso do Modelo ROQ desenvolvido pelos autores Rust, Zahorik e Keiningham (1994, 1995).

Para aplicação do Modelo ROQ, foi realizada uma pesquisa junto aos clientes da empresa escolhida, aqui chamada de Beta Metalúrgica Ltda. Os resultados da fase descritiva foram analisados por meio das técnicas estatísticas de Análise Descritiva e Exploratória de Dados, Análise Fatorial, Análise de Cluster e Análise Discriminante.

A coleta de dados e as técnicas de análise de dados foram aplicadas seguindo a forma de trabalho estabelecida pelos autores Rust, Zahorik e Keiningham (1994, 1995) na elaboração do Modelo ROQ. Os pontos, identificados como "redução viável dos preços dos produtos" e "maior percepção de flexibilidade na negociação e autonomia dos vendedores", foram alvo do desenvolvimento de cinco estratégias que visam a melhorias nos processos de atendimento e fabricação dos produtos.

O cálculo do Valor ROQ foi realizado mediante a aplicação da função Valor Presente

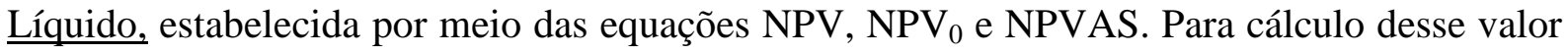
foram utilizados, como parâmetros, o investimento necessário para a viabilização das estratégias de melhorias, a margem média de contribuição do cliente, o custo de capital, a taxa de crescimento de mercado, dentre outros. Devido à falta de informação de alguns parâmetros, foram propostos dois cenários que consideraram, em primeiro lugar, as expectativas de aumento da base de clientes e da margem média de contribuição (cenário 1) e, em segundo lugar, a expectativa de aumento apenas da base de clientes (cenário 2).

Os valores ROQ aplicados aos dois cenários propostos nesta pesquisa apontaram para 
um retorno financeiro positivo, considerando-se o aumento da satisfação e intenção de recompra dos clientes da Beta Metalúrgica Ltda, mediante a realização dos investimentos em estratégias para melhorias nos processos-chave dessa empresa. Entretanto algumas limitações, encontradas no decorrer desta pesquisa, compõem uma série de sugestões para novas aplicações desse método. Essas sugestões encontram-se listadas a seguir:

- Em primeiro lugar, sugere-se, para novas aplicações do Modelo ROQ, um estudo mais elaborado sobre as formas de mensuração da satisfação já existentes nas literaturas de marketing e do comportamento do consumidor;

- Em segundo lugar, sugere-se um estudo mais aprofundado sobre a intenção de recompra e a lealdade dos clientes;

- A terceira sugestão refere-se ao método estatístico aplicado para análise dos dados. Nesta pesquisa utilizou-se a análise discriminante para estimar o impacto da satisfação para a intenção de recompra dos clientes da Beta Metalúrgica Ltda. Como sugestão para novos trabalhos, tem-se o uso da análise de regressão logística como forma de se mensurar esse impacto, dado o uso da técnica de análise de regressão no modelo original de Rust, Zahorik e Keiningham (1994, 1995).

A aplicação do Modelo ROQ na empresa Beta Metalúrgica Ltda mostrou que esse método pode ser uma ferramenta poderosa para avaliar o retorno de investimentos em qualidade sob a ótica do marketing de relacionamento. A realização desta pesquisa reforça a viabilidade da aplicação de métodos para mensuração de retorno de ações dentro da área de marketing. Por outro lado, têm-se, como fatores limitantes, os seguintes aspectos:

- A primeira limitação faz referência à forma escolhida para mensuração da satisfação dos clientes. Este estudo baseou-se somente no uso da pesquisa exploratória, sugerida pelo modelo ROQ, como forma de se dimensionarem as variáveis que envolvem a satisfação do cliente. Neste caso, um estudo mais elaborado sobre satisfação poderia ser utilizado, considerando-se a vasta literatura existente sobre esse tema e a existência de métodos já prontos para mensuração da satisfação;

- Na segunda limitação encontram-se os resultados encontrados por meio da aplicação da pesquisa quantitativa. Da mesma forma que no exemplo aplicado pelos autores Rust, Zahorik e Keiningham (1995), na rede de hotéis Nacional, o grupo de clientes insatisfeitos, que possam vir a deixar de ser clientes, foi muito pequeno, limitando-se os efeitos dos esforços de melhoria em qualidade no processo de migração desses clientes; 
- Em outra limitação estão as dificuldades encontradas para a definição das variáveis que compõem as equações necessárias para cálculo do valor ROQ. Em alguns casos, no texto dos autores Rust, Zahorik e Keiningham (1995), não se delimita, de maneira clara, a dimensão das variáveis envolvidas no processo de cálculo das equações.

Vale ressaltar que as limitações aqui encontradas não impedem a aplicação desse modelo em outros estudos. As limitações servem para identificar oportunidades para melhorias em novas aplicações do modelo ROQ.

\section{Referências}

ANDERSON, E. W.; FORNELL, C.; LEHMANN, D. R. Customer Satisfaction, Market Share, and Profitability: findings from Sweden. Journal of Marketing, v. 58, p. 53-67, jul. 1994.

BABBIE, E. Métodos de Pesquisas de Survey. Belo Horizonte: Editora UFMG, 2001.

CHARNET, R.; FREIRE, C. A.; CHARNET, E. M. R.; BONVINO, H. Análise de Modelos de Regressão Linear com Aplicações. Campinas: Ed. Unicamp, 1999.

COVER, J. Profitability analysis - a necessary tool for success in the $21^{\text {st }}$ Century. ABA Banking Journal, v. 91, n. 2, p. 78, fev. 1999.

DANTAS, E. B. A Informação como Insumo da Prática do Marketing na Gestão do Conhecimento do Cliente. Universidade de Brasília. BOCC - Biblioteca On-Line de Ciências da Comunicação, 2006. Disponível em:

<http://www.bocc.ubi.pt/_esp/autor.php?codautor=923>. Acesso em: 30 maio 2006.

HAIR Jr., J. F.; ANDERSON, R. E.; TATHAM, R. L.; BLACK, W. C. Análise Multivariada de Dados. 5. ed. Porto Alegre: Bookman, 2005b.

HAIR Jr., J.F.; BABIN, B.; MONEY, A. H.; SAMOUEL, P. Fundamentos de Métodos de Pesquisa em Administração. Porto Alegre: Bookman, 2005a.

MALHOTRA, N. K. Pesquisa de Marketing: uma orientação aplicada. 3. ed. Porto Alegre: Bookman, 2001.

MORGAN, R. M.; HUNT, S. D. The Commitment-Trust Theory of Relationship Marketing. Journal of Marketing, v. 58, p. 20-38, jul. 1994.

MULHERN, F. J. Costumer profitability analysis: measurement, concentration and research directions. Journal of Interactive Marketing, v. 13, n. 1, p. 25-40, 1999.

RAVALD, A.; GRÖNROOS, C. The value concept and relationship marketing . European Journal of Marketing, v. 30, n. 2, p. 19-30, 1996. 
RIBEIRO, A. H. P.; VEIGA, R. T.; SOUZA, D. V.; REIS, A. F.; ROCHA, M. N.;

MARTINS, F. F. Retorno Financeiro dos Investimentos em Marketing: Uma Aplicação do Modelo ROQ. Anais Eletrônicos XXIX Enanpad, Brasília, p. 1-16, 2005.

RUST, R. T.; KEININGHAM, T. L; CLEMENS, S.; ZAHORIK, A. Return on Quality at Chase Manhattan Bank. Interfaces, v. 29, n. 2, p. 62-72, mar./abr. 1999.

RUST, R. T.; ZAHORIK, A. J.; KEININGHAM T. L. Mensurando o Impacto Financeiro de sua Empresa. Rio de Janeiro: Qualitymark, 1994.

RUST, R. T.; ZAHORIK, A. J.; KEININGHAM, T. L. Return on Quality (ROQ): Making Service Quality Financially Accountable. Journal of Marketing, v. 59, p. 58-70, abr. 1995. 